Received 00th January 2020, Accepted 00th January 2020 DOI: $10.1039 / x 0 \times x 00000 x$

\title{
Twisted Bodipy Derivative: Intersystem Crossing, Electron Spin Polarization and Application As a Novel Photodynamic Therapy Reagent
}

\author{
Yu Dong, $\ddagger^{\mathrm{a}}$ Prashant Kumar, $\ddagger^{\mathrm{b}}$ Partha Maity, $\ddagger^{\mathrm{c}}$ Ivan Kurganskii, ${ }^{\mathrm{d}}$ Shujing $\mathrm{Li},{ }^{\mathrm{e}}$ Ayhan Elmali, ${ }^{\mathrm{f}}$ \\ Jianzhang Zhao, ${ }^{*^{\mathrm{a}}}$ Daniel Escudero, ${ }^{{ }^{\mathrm{b}}}$ Huijian Wu, ${ }^{\mathrm{e}}$ Ahmet Karatay ${ }^{{ }^{\mathrm{f}}}$, Omar F. Mohammed ${ }^{{ }^{\mathrm{c}}}$ and \\ Matvey Fedin* ${ }^{d}$
}

\begin{abstract}
The photophysical properties of a heavy atom-free Bodipy derivative with twisted $\pi$-conjugation framework were studied. Efficient intersystem crossing (ISC. Quantum yield: 56\%) and exceptionally long-lived triplet state wereobserved (4.5 ms. In solid polymer film matrix; the intrinsic triplet state lifetime in fluid solution is $197.5 \mu \mathrm{s})$. Time-resolved electron paramagnetic resonance (TREPR) spectroscopy and DFT computations confirmed the delocalization of triplet state on whole twisted $\pi$ conjugation framework, and the zero-field-splitting (ZFS) $D$ parameter is $D=-69.5 \mathrm{mT}$, which is smaller than the 2,6diiodoBodipy $(D=-104.6 \mathrm{mT}$ ). The electron spin polarization (ESP) phase pattern of the triplet state TREPR spectrum of the twisted Bodipy is ( $a, a, e, a, e, e)$, which is different from that of 2,6-diiodoBodipy $(e, e, e, a, a, a)$, indicates the electron spin selectivity of the ISC of the twisted structure is different from that of the spin orbital coupling effect. The computed spinorbit coupling matrix elements $\left(0.154 \sim 1.964 \mathrm{~cm}^{1}\right)$, together with the matched energy of the $\mathrm{S}_{1} / \mathrm{T}_{\mathrm{n}}$ states, the ISC was proposed to be via $S_{1} \rightarrow T_{2} / T_{3}$. The computational results are in agreement with the TREPR results on the electron spin selectivity (the over population of the $T_{Y}$ sublevels of the $T_{1}$ state). The advantage of long-lived triplet state of twisted Bodipy was demonstrated by the efficient singlet oxygen $\left({ }^{1} \mathrm{O}_{2}\right)$ photosensitizing $\left(\Phi_{\Delta}=50.0 \%\right)$ even under severe hypoxia atmosphere $\left(p \mathrm{O}_{2}=0.2 \%, \mathrm{v} / \mathrm{v}\right)$. High light toxicity $\left(E C_{50}=1.0 \mu \mathrm{M}\right)$ and low dark toxicity $\left(\mathrm{EC}_{50}=78.5 \mu \mathrm{M}\right)$ was observed for the twisted Bodipy, cellular studies demonstrate its potential as a novel potent heavy atom-free photodynamic therapy (PDT) reagent.
\end{abstract}

\section{Introduction}

Triplet photosensitizers (PSs) are widely used in photocatalysis, ${ }^{13}$ photon upconversion, ${ }^{4}{ }^{-6}$ and photodynamic therapy (PDT), ${ }^{714}$ due to the efficient triplet state generation ability. The fundamental photophysical processes involved in the triplet PSs and their applications include ISC, energy transfer or electron transfer. ${ }^{15}$ However, ISC is a spin forbidden nonradiative transition, which is usually difficult to occur for the

\footnotetext{
State Key Laboratory of Fine Chemicals, School of Chemical Engineering, Dalian University of Technology, E-208 West Campus, 2 Ling Gong Road, Dalian 116024, China.E-mail: zhaojzh@dlut.edu.cn

b. Department of Chemistry, KU Leuven, Celestijnenlaan 200F, B-3001 Leuven, Belgium. E-mail: Daniel.escudero@kuleuven.be

Division of Physical Sciences and Engineering, King Abdullah University of Science and Technology (KAUST), Thuwal 23955-6900, Kingdom of Saudi Arabia. E-mail: omar.abdelsaboor@kaust.edu.sa

d. International Tomography Center, SB RAS Institutskaya Str., 3A, and Novosibirsk State University, Pirogova str. 2, 630090 Novosibirsk, Russia. E-mail: mfedin@tomo.nsc.ru

${ }^{e}$. School of Bioengineering \& Key Laboratory of Protein Modification and Disease, Liaoning Province, Dalian University of Technology, Dalian, China. E-mail: wuhj@dlut.edu.cn

f. Department of Engineering Physics, Faculty of Engineering, Ankara University, 06100 Beşevler, Ankara, Turkey.E-mail: Ahmet.Karatay@eng.ankara.edu.tr

\pm These authors contributed equally to this work.

$\dagger$ Electronic Supplementary Information (ESI) available: [Steady state and femtosecond/nanosecond transient absorption spectra, and PDT studies with cells]. See DOI: $10.1039 / x 0 x x 00000 x$
}

aromatic chromophores with planar $\pi$-conjugation framework. The ISC rate can be described by simplified equation (Eq. 1), the rate is proportional to spin orbital coupling (SOC) matrix element and inversely proportional to energy gap between $\mathrm{S}_{\mathrm{m}} / \mathrm{T}_{\mathrm{n}}$ states. $^{16}$

$$
k_{\mathrm{ISC}} \propto \frac{\left\langle\mathrm{T}_{\mathrm{n}}\left|H_{S O}\right| \mathrm{S}_{\mathrm{m}}\right\rangle^{2}}{\left(\Delta E_{\mathrm{S}_{\mathrm{m}}-\mathrm{T}_{\mathrm{n}}}\right)^{2}}
$$

For instance, the efficient ISC of anthracene is due to the small energy gap between $S_{1}$ and $T_{2}$ state. ${ }^{17}$ However, the delicate energy matching of the $S_{1} / T_{n}$ states is easy to be influenced by substituent and difficult to predict. The ISC quantum yield of anthracene decreases from 0.73 to less than 0.05 for anthracene derivatives, e.g. 9,10-diphenylanthracene (DPA), due to the subtle energy shift of $T_{2}$ state by the introduction of phenyl substitution. ${ }^{17,18}$

Heavy atom effect (HAE) is the most common strategy to enhance the SOC by attaching transition metal atoms ( $\mathrm{Pt}, \mathrm{Ir}, \mathrm{Ru}$, etc) or halogen atoms $(\mathrm{Br}, \mathrm{I})$ to a chromophore. ${ }^{5,14,15,19}$ Due to the high cost of the precious metals and the biotoxicity of halogen atoms, heavy atom-free triplet PSs have attracted much attention in recent years. Concerning this aspect, $\mathrm{n}$ $\pi^{*} \leftrightarrow \pi-\pi^{*}$ transition, ${ }^{20}{ }^{22}$ electron spin converter, ${ }^{23}{ }^{26}$ radicalenhanced ISC, ${ }^{27,28}$ exciton coupling, ${ }^{29,30}$ singlet fission ${ }^{31}$ and 
charge recombination (CR)-induced $\mathrm{ISC}^{32}{ }^{35}$ are promising methods to attain efficient ISC in heavy atom-free chromophores. However, some drawbacks exist for these methods, for instance, the complicated synthesis procedures.

We notice that ISC was observed in hydrocarbon compounds with twisted $\pi$-conjugation structures, such as tolanes and helicene. ${ }^{3638}$ For the helicenes, SOC between two $\pi-\pi^{*}$ states increases as compared to that in coplanar molecules, as the removal of the mirror-plane symmetry of the twisted molecule allows nonvanishing matrix elements associated with each molecular orbital angular momentum component. ${ }^{37}$ Moreover, the SOC matrix elements become sensitive to the spatial extension of the molecular wave function in the out-of-plane direction. The spin-orbital coupling matrix elements become larger with more distribution of the triplet wave function on the twisted moiety. ${ }^{37}$ Recently, ISC of twisted structures of some traditional $\pi$-conjugation planar chromophores were found, such as porphyrins, perylene-3,4,9,10-bis(dicarboximide) (also abbreviated as perylenebisimide, PBI) and bodipy. ${ }^{39}{ }^{45}$ However, the study of photophysical processes and ISC mechanism of these twisted molecules was rare. ${ }^{41,42,44}$ Moreover, the molecular structure diversity needs to be fully explored for this novel method to enhance ISC. Therefore, the reported bodipy derivative (BDP-1, Scheme 1) was selected as the target compound. The twisted structure of BDP-1 was confirmed by single crystal X-ray diffraction and DFT calculation in the previous studies. ${ }^{46}$ However, only basic photophysical properties (UV-Vis absorption and fluorescence) were studied. ${ }^{46}$ Recently, the triplet state properties and the application of BDP-1 in triplet-triplet annihilation upconversion (TTA-UC) were reported by our group. ${ }^{47}$ In this manuscript, we will focus on the study of the detail photophysical process, ISC kinetics, electron spin selectivity of the ISC, the spatial confinement of the triplet state wave function and the applications of BDP-1 in PDT.

In this work, the photophysical and photochemical properties of Bodipy with twisted $\pi$-conjugation structure (BDP-1, Scheme 1) have been studied using steady state and femtosecond, subnanosecond and nanosecond transient optical spectroscopic methods. The electron spin selectivity of the ISC and spatial confinement of the triplet state wave function and the were studied with pulsed laser excited electron paramagnetic resonance (TREPR) spectroscopy. The twisted Bodipy derivative is also applied as a novel PDT reagent, high light toxicity and low dark toxicity were observed.

\section{Results and Discussion}

\section{Molecular structures}

he Bodipy derivative BDP-1 (Scheme 1) was previously reported with twisted $\pi$-conjugation framework. ${ }^{46}$ Recently, we discovered the efficient ISC of this compound. ${ }^{47}$ Therefore, it is worthy to study the detailed photophysical properties to attain in-depth understanding of ISC enhanced by twisted structure. The conventional triplet PSs 2,6-diiodoBodipy (BDP-2) and 2,6-
diiodobisstyrylBodipy (BDP-3) containing heavy atoms were used as reference in the studies.

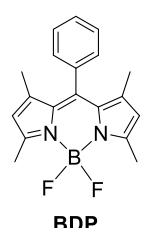

BDP

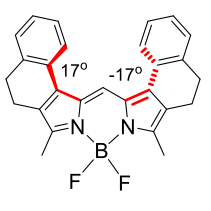

BDP-1

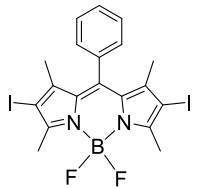

BDP-2

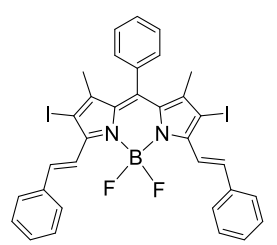

BDP-3
(2,6-diiodoBodipy) (2,6-diiodobisstyrylBodipy)

Scheme 1 Molecular structures of BDP-1 and the reference compounds. The dihedral angles of BDP-1 were determined by single crystal X-ray diffraction. ${ }^{46}$

\section{UV-visible absorption and fluorescence spectra}

The UV-vis absorption spectra of the compounds were studied (Fig. 1). For BDP-1, the absorption band centered at $564 \mathrm{~nm}$ is assigned to the $S_{0} \rightarrow S_{1}$ transition (refer to the later DFT computation section), whereas the weaker broad absorption band in the range $350 \mathrm{~nm}$ to $500 \mathrm{~nm}$ is attributed to $S_{0} \rightarrow S_{2}$ and $\mathrm{S}_{0} \rightarrow \mathrm{S}_{3}$ transitions. The absorption of BDP-1 is red-shifted as compared to BDP and BDP-2 and blue-shifted than BDP-3 (Table 1). However, the absorption is stronger than all the reference compounds (molar absorption coefficient $\varepsilon=1.2 \times 10^{5} \mathrm{M}^{-1} \mathrm{~cm}^{-1}$ at $564 \mathrm{~nm}$ for BDP-1, $\varepsilon=7.80 \times 10^{4} \mathrm{M}^{-1} \mathrm{~cm}^{-1}$ at $500 \mathrm{~nm}$ for BDP, $\varepsilon=7.50 \times 10^{4} \mathrm{M}^{-1} \mathrm{~cm}^{-1}$ at $533 \mathrm{~nm}$ for BDP-2 and $\varepsilon=8.50 \times 10^{4}$ $\mathrm{M}^{1}{ }^{1} \mathrm{~cm}^{-1}$ at $638 \mathrm{~nm}$ for BDP-3, respectively. Table 1$)$, which is an advantage for further applications. For the UV-Vis absorption spectra in polymer film (Clear Flex 50॰, CLRFLX), ${ }^{48}$
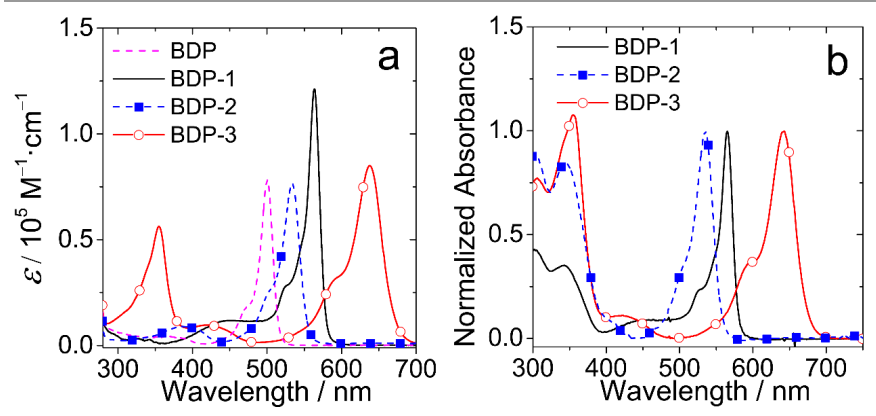

Fig. 1 UV-Vis absorption spectra (a) BDP, BDP-1, BDP-2 and BDP-3 in DCM. $\varepsilon$ is the molar absorption coefficient. (b) Normalized absorption spectra of BDP-1, BDP-2 and BDP-3 in Clear Flex 50, film. $20^{\circ} \mathrm{C}$.
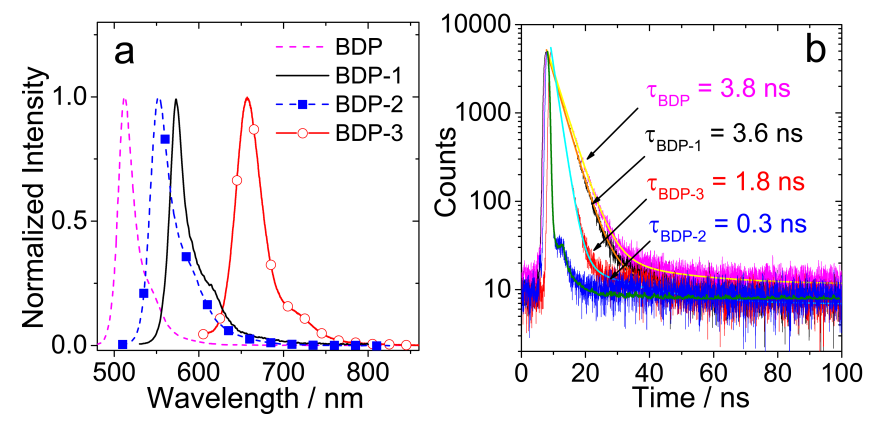

Fig. 2 Fluorescence spectra in DCM (a) Normalized fluorescence emission spectra of BDP, BDP-1, BDP-2 and BDP-3; (b) Fluorescence decay traces of BDP at $520 \mathrm{~nm}$ (blue), BDP-1 at $572 \mathrm{~nm}$ (black), BDP-2 at $550 \mathrm{~nm}$ (magenta) and BDP-3 at $660 \mathrm{~nm}$ (orange), $c=1.0 \times$ $10^{5} \mathrm{M} .20^{\circ} \mathrm{C}$ 

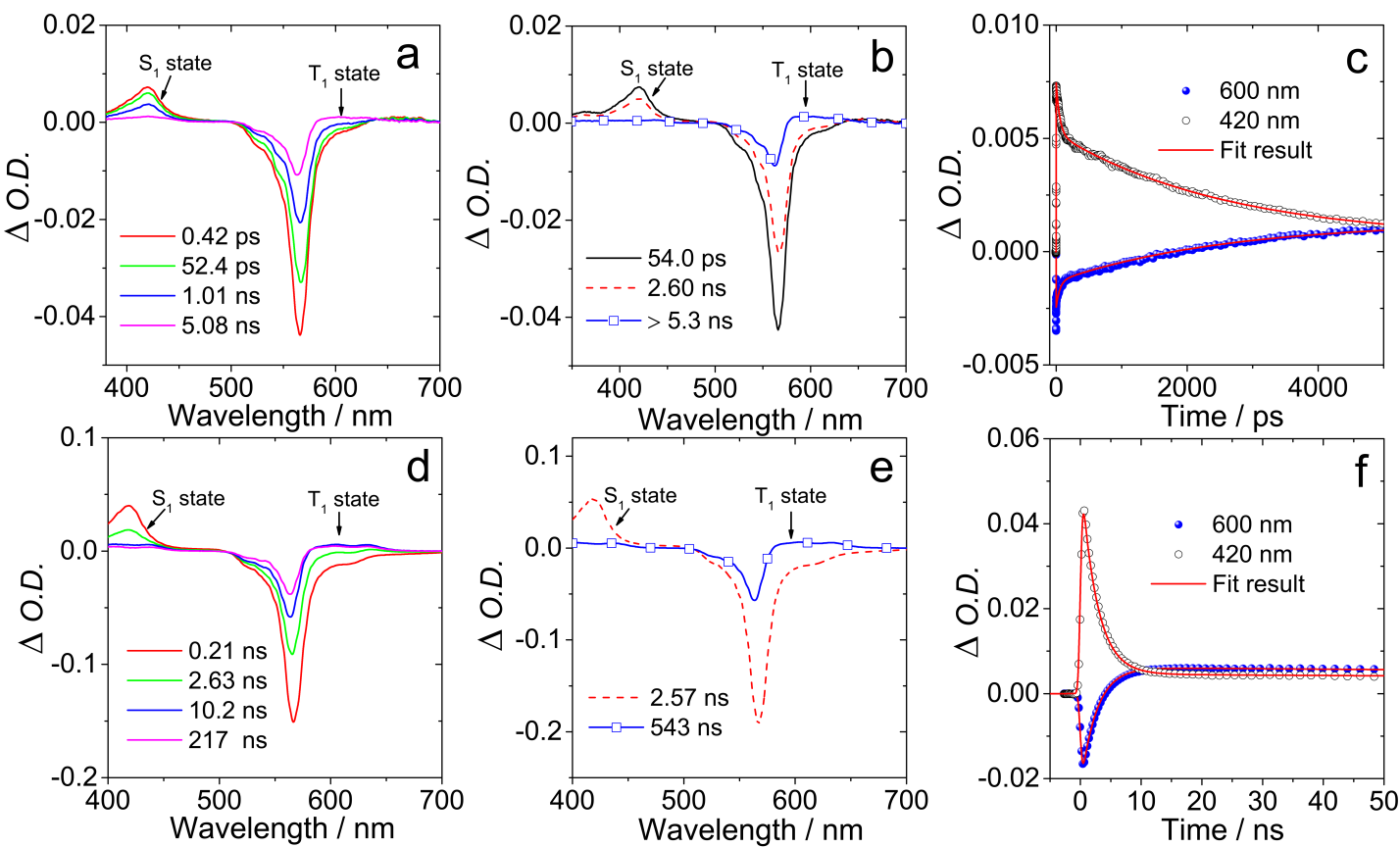

Fig. 3 Femtosecond transient absorption (fs-TA) spectra of BDP-1. (a) fs-TA spectra at different time delays. (b) Evolution-associated difference spectra (EADS) obtained by the global fitting of the data in (a). (c) Kinetics at selected wavelengths. (d) Sub ns-TA spectra at different time slots. (e) Evolution-associated difference spectra (EADS). (f) Kinetics at selected wavelengths. EADS were obtained by singular value decomposition (SVD) and global fitting (sequential model). $\lambda_{\mathrm{ex}}=540 \mathrm{~nm}, \lambda_{\text {probe }}=420$ and, 600 $\mathrm{nm}, \mathrm{c}=1.0 \times 10^{-5} \mathrm{M}$ in DCM, $20^{\circ} \mathrm{C}$.

the absorption shows no obvious change compared with that in fluid solution. In contrast, for the absorption spectra of compounds in casting films (prepared by evaporation of DCM solution. Fig. S1†), broader absorption bands were obtained, which is due to aggregation. Therefore, we conclude that no obvious aggregation exists in the Clear Flex 50" films.

The fluorescence spectra of the compounds were studied (Fig. 2). The emission band of BDP-1 is centred at $570 \mathrm{~nm}$, and show no significant solvent polarity-dependency (Fig. S2a†). The fluorescence spectra were also measured in polymer film (Fig. $\mathrm{S} 2 \mathrm{~b} \dagger$ ). For BDP-2, the fluorescence peaks are slightly broader than the spectra in solutions, which may be due to aggregation in film. For BDP-1 and BDP-3, the emission shows no obvious change compared with the spectra in solution, indicating no aggregation in films. The absolute fluorescence quantum yields $\left(\Phi_{\mathrm{F}}\right)$ were determined (Table 1 and Table $\mathrm{S} 1 \dagger$ ). The highest fluorescence quantum yield was observed in toluene as $43 \%$ and the lowest is in DCM as $31 \%$, which are both smaller than the reported values $(50 \% \sim 54 \%)$. It may be due to the experimental condition (absolute fluorescence quantum yield was determined by optical integrating sphere in this article, and relative fluorescence quantum yield was determined by using standard compound in the previous work). ${ }^{46}$ The time-resolved emission spectra were also measured (Fig. $2 \mathrm{~b}$ and Fig. $\mathrm{S} 3 \dagger$ ). The fluorescence lifetime $\left(\tau_{\mathrm{F}}\right)$ of BDP-1 is $3.6 \mathrm{~ns}$ (mono-exponential decay) in DCM, which is similar to the $\tau_{F}$ value of BDP (3.8 ns) and it is independent on solvent polarity. ${ }^{46}$

\section{Ultrafast transient absorption spectroscopy}

Femtosecond transient absorption (fs-TA) and sub-nanosecond transient absorption (sub-ns-TA) spectroscopies were used to determine the excited state dynamics of BDP-1 (Fig. 3), especially the rate constant of ISC.

The fs-TA spectra were measured in DCM (Fig. 3a). An intensive negative band centered at $565 \mathrm{~nm}$ was observed immediately upon excitation at $540 \mathrm{~nm}$. The wavelength was in agreement with the steady-state absorption spectra (Fig. 1a), which can be attributed to the depletion of the ground state, i.e. this is the ground state blenching (GSB) band. Another weak negative band localized at the region of 590 650 $\mathrm{nm}$ was observed, which is attributed as the stimulated emission (SE) band. Moreover, an excited state absorption (ESA) band centered at $420 \mathrm{~nm}$ was also observed, which is assigned to the absorption of $S_{1} \rightarrow S_{n}$ transitions. The weak ESA band was observed in the region of 590 650 nm, which is the characteristic signal of triplet state of BDP-1 (Fig. 3a). ${ }^{47}$ The triplet state signal increased alongwith the decreasing of the ESA band of $S_{1} \rightarrow S_{n}$ transitions at $420 \mathrm{~nm}$. The lasting of the GSB on 5.3 ns time scale of measurement also indicate the existence of long-lived species. Therefore, we conclude that the ISC process occurs.

Evolution-associated difference spectra (EADS) were obtained by global fitting in the sequential model to determine the rate constants of photophysical process (Fig. 3b). The species with 54.0 ps lifetime can be attributed to the FranckCondon $\mathrm{S}_{1}$ state, its structural relaxation and solvent relaxation take $54.0 \mathrm{ps}$. The species with lifetime of $2.60 \mathrm{~ns}$ is assigned to the relaxed $S_{1}$ state. The long-lived species with characteristic of $T_{1} \rightarrow T_{n}$ transitions is assigned to the triplet state signal of BDP-1, supported by reported ns TA results. ${ }^{47}$ Therefore, the time constant of ISC is determined as $2.60 \mathrm{~ns}$, which is slightly shorter than the fluorescence lifetime (3.6 ns). 
Table 1 The photophysical properties of the compounds

\begin{tabular}{|c|c|c|c|c|c|c|c|}
\hline & $\lambda_{\mathrm{abs}}{ }^{a}\left(\varepsilon^{b}\right)$ & $\lambda_{\mathrm{F}}{ }^{c}$ & $\Phi_{F}^{d}$ & $\tau_{\mathrm{F}}^{e}$ & $\Phi_{\Delta}^{f}$ & $\Phi_{\mathrm{T}}{ }^{g}$ & $\boldsymbol{\tau}_{\top}^{h}$ \\
\hline BDP-1 & $564(1.21)$ & 572 & $0.31^{i} / 0.29^{j}$ & 3.6 & $0.55^{i} / 0.59^{j}$ & 0.56 & 4.5 \\
\hline BDP-2 & $533(0.75)$ & 552 & $0.027^{k}$ & 0.3 & $0.92^{k}$ & -1 & 0.7 \\
\hline
\end{tabular}

${ }^{a} c=1.0 \times 10^{5} \mathrm{M}$, in nm. ${ }^{b}$ Molar absorption coefficient. $\varepsilon$ values are in $10^{5} \mathrm{M}^{1} \mathrm{~cm}^{1}{ }^{1}{ }^{c}$ Fluorescence emission maxima wavelength, in nm. ${ }^{d}$ Absolute fluorescence quantum yield determined by optical integrating sphere, error: $\pm 0.01 .{ }^{e}$ Fluorescence lifetime, $\lambda_{\text {ex }}=510 \mathrm{~nm}$, in ns, $c=1.0 \times 10^{5} \mathrm{M}$. ${ }^{f}$ Singlet oxygen quantum yield $\left(\Phi_{\mathrm{A}}\right)$, Ru(bpy) ${ }_{3}$ as standard $\left(\Phi_{\Delta}=0.57\right.$ in $\left.A C N\right)$ when excited into the $S_{n}$ state $(n \geq 2)$. 2,6-diiodobodipy was used as standard $\left(\Phi_{\Delta}=0.85\right.$ in toluene) when excited into the $S_{1}$ state. Estimated

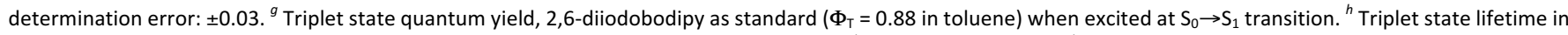
Clear Flex 50: film, in ms. ${ }^{i}$ Excited at $S_{0} \rightarrow S_{1}$ transition. ${ }^{j}$ Excited at $S_{0} \rightarrow S_{n}$ transition $(n \geq 2) .{ }^{k}$ Literature results. ${ }^{49,50 ~}{ }^{\prime}$ Not determined.

Since the ISC is slow, the sub-ns-TA spectra were also studied (Fig. 3d). An obvious negative band at the region of 500 650 $\mathrm{nm}$ was observed upon excitation (Fig. 3d), which is attributed to the the convolution of ground state blenching band (GSB) and stimulated emission (SE) band. A positive band at $420 \mathrm{~nm}$ assigned to ESA of $S_{1} \rightarrow S_{n}$ transient was also observed and same as the results obtained in fs TA (Fig. 3a), indicating the species is $S_{1}$ state. The increase of ESA band of $T_{1} \rightarrow T_{n}$ transient as 600 $\mathrm{nm}$ was clearly observed. The time constant of ISC was also determined as $2.57 \mathrm{~ns}$ by EADS of sub-ns-TA (Fig. 3e), which is similar as the value obtained in fs TA $(2.60 \mathrm{~ns})$. It should be pointed out that the triplet state in sub- ns-TA spectra was short-lived (543 ns, Fig. 3e) due to the aerated conditions used in the measurement. Therefore, the triplet state lifetime of BDP-1 is much short than our reported result $(197.5 \mu \mathrm{s}) .{ }^{47}$ Thus, we can conclude that the triplet state of BDP-1 was produced by ISC process with 2.60 ns time constant.

\section{Nanosecond transient absorption spectroscopy: triplet state properties of the compounds in polymer film}

The nanosecond transient absorption (ns-TA) spectra and triplet state lifetime of BDP-1 in fluid solution have been reported, the intrinsic triplet state lifetime was determined as $197.5 \mu \mathrm{s}$ in DCM. ${ }^{47}$ However, the triplet state may be with different lifetime in different matrices. It is a promising method to suppress the molecular vibration and the collision by doping triplet PSs into amorphous polymer matrices to prolong the triplet state lifeitmes. ${ }^{51}$. 33 Inspired by the strategy, we mixed the DCM solution of triplet PSs and the commercially available polyurethane precursors (Clear Flex 50, CLRFLX) $^{48}$ together to prepare the flexible material and the triplet state properties were investigated by ns-TA spectroscopy (Fig. 4). For the heavy atom-free triplet PS BDP-1, the characteristic ESA bands of BDP1 were observed, which are same as the characteristic signals in fluid solutions. ${ }^{47}$ More importantly, an exceptionally long-lived triplet state was obtained $(4.5 \mathrm{~ms})$, which is 20 -fold longer than the intrinsic triplet state lifetime in solution (197.5 $\mu \mathrm{s}) .{ }^{47}$ The molecular motion and vibration are both restricted in solid polymer matrices and these factors should be responsible for the long-lived triplet state. In addition, the triplet state lifetime is also much longer than the value of iodo-subsitued Bodipy and styrylBodipy derivatives under similar conditions. For BDP-2, the triplet state lifetime was determined as $0.7 \mathrm{~ms}$ (Fig. S10†), which is similar as the room temperature phosphorescence lifetime in polymer matrix, ${ }^{53}$ and much longer than the intrinsic triplet state lifetime in solution $(276 \mu \mathrm{s}) .{ }^{54}$ Note, the lifetime of BDP-2 in film is also much shorter than BDP-1.
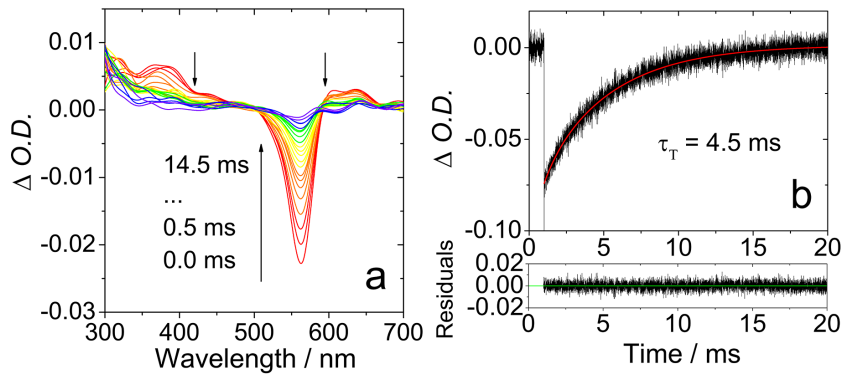

Fig. 4 (a) Nanosecond transient absorption spectra of BDP-1 doped Clear Flex 50* film under $\mathrm{N}_{2}$ atmosphere. (b) Decay trace at $560 \mathrm{~nm}$. $\lambda_{\mathrm{ex}}=550 \mathrm{~nm}, 17.6 \mu \mathrm{mol} / \mathrm{g}$ (BDP-1/Clear Flex $\left.50^{\circ}\right), 20^{\circ} \mathrm{C}$.

For the diiodostyrylBodipy BDP-3, the ESA bands are consistent with the characteristic signal in fluid solution, ${ }^{49}$ indicating the identical triplet state was obtained in film (Fig. S11 $\uparrow$ ). The triplet state lifetime was determined as $1.7 \mu \mathrm{s}$ and $1.8 \mu \mathrm{s}$ in film and solution, respectively (Fig. S11b $\dagger$ ). ${ }^{50}$ The short lifetime indicates the HAE to reduce the triplet state lifetime for styrylBodipy chromophore. The triplet state lifetime of heavy atom-free triplet PSs is longer due to the elimination of HAE. Furthermore, the lifetime is exceptionally longer in solid film compared with that in fluid solution. Therefore, this may become an advantage in further application by doping triplet PSs into nanoparticles or other solid materials in PDT, because it was shown that triplet PSs with longer triplet state lifetime are efficient in the hypoxia microenvironment of the tumour tissue.

Time-resolved electron paramagnetic resonance spectroscopy (TREPR): electron spin selectivity of the twisting-induced ISC and the spatial confinement of the triplet state wave function

Transient optical absorption/emission spectroscopies give the information on the electronic excited states and the electronic transitions between the states, however, the confinement of the triplet wave function and the electron spin selectivity of the ISC can't be unraveled directly by the optical spectroscopy. However, the confinement of the two unpaired electrons of the triplet state, or the average distance, can be manifested by the magnetic dipole-dipole interaction, i.e. the Zero-Field Splitting (ZFS) and the corresponding parameters $D$ and $E$. The ZFS parameter can be influenced by spin-spin coupling and SOCs, however, the SOCs normally show a dominant contribution in 
the case of transition metal complexes. The influence of spinspin coupling is dominant for organic compounds. ${ }^{55,56}$ The ZFS parameter $D$, which can be directly measured by TREPR spectroscopy, ${ }^{55,57}$ depends on the magnitude of the dipole interaction. In the case that the spin-orbit interaction is small enough, $D$ provides a good estimation of the distance between the two electrons, based on the point dipole approximation. Moreover, the electron spin polarization (ESP) of the spin selective ISC can be also unraveled with the TREPR spectra, which gives information on the ISC mechanisms and the population rates of the three sublevels of the $T_{1}$ state. ${ }^{55,5864}$

The TREPR spectrum was recorded by excitation of the frozen solution (at $80 \mathrm{~K}$ ) of BDP-1 in toluene/MeTHF (3:1, v/v) with 532 $\mathrm{nm}$ pulsed laser (Fig. 5). The magnitude of the parameter $D$ can be used to evaluate the localization of triplet state and ZFS parameter $E$ indicates the symmetry of the compound at the $T_{1}$ state. The $D$ value of BDP-1 $(-69.5 \mathrm{mT})$ is noticeably smaller than that in BDP-2 (-104.6 mT, Table 2). Moreover, the $D$ value of BDP-1 is also smaller than that in heavy atom-free Bodipy derivatives (ca. $-82 \mathrm{mT}$ ), which are without extended $\pi$ conjugation framework. ${ }^{65,66}$ Most reasonably, this indicates that the unpaired electrons of the triplet state in BDP-1 are with larger separation, i.e. more delocalized on the $\pi$-conjugated framework. Note that, in principle, SOC due to the twisted structure of BDP-1 molecule can also contribute to the observed reduction in $D$. The decreasing of the $D$ parameter is also in agreement with the spin density surface analysis, which indicates the partial delocalization of spin density to the overall $\pi$-conjugated system, although the localization on Bodipy chromophore is dominant (refer to a later section). ${ }^{46}$ Moreover, the distribution of the two unpaired electrons on the whole molecule is in agreement with the ISC mechanism, which is supposed to be enhanced by the twisted $\pi$-conjugation frame of BDP-1. ${ }^{37,41,42,44}$ The $E$ parameter mainly demonstrate the rhombicity of the triplet wave function. ${ }^{40,55,57}$ The magnitude of E parameter of BDP-1 is smaller than that of BDP-2 (-15.5 mT vs $-22.8 \mathrm{mT}$, Table 2). For BDP-1, the rhombicity of the triplet wave function, indicated by the spin density surface, is less
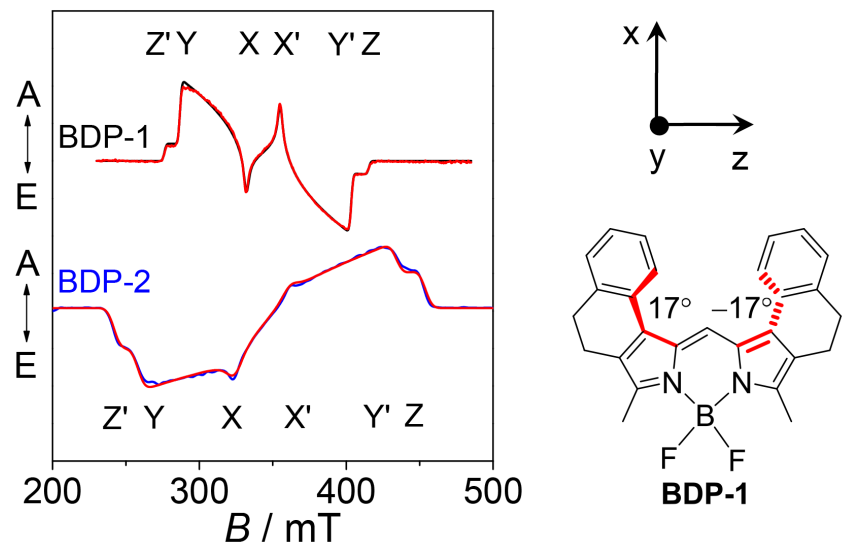

Fig. 5 TREPR spectra of BDP-1 and BDP-2. The simulations (red lines) of the compounds are also shown. Spectrum was recorded by excitation of the frozen solution with $532 \mathrm{~nm}$ nanosecond pulsed laser. In toluene/MeTHF $(3: 1, \mathrm{v} / \mathrm{v}) .80 \mathrm{~K}$. Integration time window: 0.0 1.8 $\mu \mathrm{s}$. The dihedral angles of BDP-1 were determined by single crystal X-ray diffraction. ${ }^{46}$
Table 2 ZFS parameters ( $D$ and $E$ ) and relative population rates $P_{x, y, z}$ of the zero-field spin states of compounds

\begin{tabular}{ccccc}
\hline Compound & $D(\mathrm{mT})$ & $E(\mathrm{mT})$ & $P_{x}: P_{y}: P_{z}^{a}$ & $\begin{array}{c}\Delta P=\left|P_{x}-P_{y}\right| / \\
\left|P_{y}-P_{z}\right|^{b}\end{array}$ \\
\hline BDP-1 & -69.5 & -15.5 & $0.00: 1.00: 0.21$ & 1.27 \\
BDP-2 & -104.6 & -22.8 & $0.20: 0.00: 1.00$ & 0.20
\end{tabular}

${ }^{a}$ Representative population of the zero-field spin states normalized to $1 .{ }^{b}$ Unique ratios of populations, which determine the ESP.

significant than BDP-2, therefore, the smaller $E$ parameter observed for BDP-1 as compared to BDP-2 is reasonable. On the other hand, the E/D value remains closely the same in BDP-1 and BDP-2, possibly implying that electron delocalization in $T_{1}$ state along $X$ axis does not change considerably.

The ESP pattern of the TREPR spectrum of the triplet state of BDP-1 was obtained as $(a, a, e, a, e, e)$ ( $a$ and $e$ represent for enhanced absorptive and emissive polarizations, respectively). The results are drastically different from featured ESP patterns via RP-ISC, i.e. $(a, e, e, a, a, e)$ or $(e, a, a, e, e, a) .{ }^{55}$ Internestingly, the ESP pattern is also completely different from that of BDP-2 $(e, e, e, a, a, a)$, for which the ISC is enhanced by the HAE (normal spin-orbit coupling). ${ }^{57}$ The relative population rate of the three sublevels of the triplet state is $P_{x}: P_{y}: P_{z}=0.00: 1.00$ : 0.21 for BDP-1, whereas it is $P_{x}: P_{y}: P_{z}=0.20: 0.00: 1.00$ for BDP-2. The results indicate that the overpopulation is on $T_{y}$ for BDP-1, and it is $T_{z}$ for BDP-2. Note the ESP is the same to that observed with an orthoganal Bodipy dimer. ${ }^{65}$ Based on the observed ESP phase pattern of the triplet TREPR spectra, the radical pair ISC (RP-ISC) and spin orbital charge transfer ISC (SOCT-ISC) mechanisms can be excluded. Moreover, the ESP phase pattern of the TREPR spectra of BDP-1 does not change at longer delay time (Fig. S12 $\uparrow$ ), indicating there is no anisotropic depopulation of the sublevels of the $T_{1}$ state. $^{40,44}$

Compared with a recently reported twisted bodipy derivative showing ISC, the absolute value of paramenter D of BDP-1 is larger (69.5 mT v.s. $59.5 \mathrm{mT}){ }^{44}$ indicating the delocalization of triplet state wave function of BDP-1 is smaller than the recently reported twisted Bodipy showing ISC. Moreover, the previously reported twisting Bodipy derivative shows an ESP phase pattern of $(a, e, a, e, a, e)$ for the triplet TREPR spectrum, which is different from the current one. These results demonstrated the interesting electron spin chemistry of the ISC of the twisted Bodipy derivatives, i.e. the electron spin selectivity of the ISC of the twisted Bodipy derivatives can be drastically different. ${ }^{44}$

Quantum chemical investigations: optimized ground state geometry, spin orbit couplings and ISC mechanism

The ground state geometry of BDP-1 was optimized (Fig. 6). The dihedral angles between Bodipy $\pi$-conjugation system and the peripheral phenyl rings were determined as $21.4^{\circ}$. The torsion is similar to that determined with single crystal X-ray diffraction $\left(17.0^{\circ}\right) .^{46}$ In addition, the compound shows a "propeller-like" geometry, also in agreement with the single crystal molecular structure. ${ }^{46}$ The geometries of $S_{1}$ state and $T_{1}$ state were also optimized (Fig. $\mathrm{S} 13 \dagger$ ). For $\mathrm{S}_{1}$ state and $\mathrm{T}_{1}$ state, the dihedral angles were determined as $17.2^{\circ}$ and $18.8^{\circ}$, respectively, 
Table 3 COSMO-RI-ADC(2)/def2-TZVP singlet and triplet electronic transition energies (in eV) and MO contribution of BDP-1 at their optimized geometries; along with SOCs (in $\mathrm{cm}^{-1}$ ) between the Involved $T_{n}$ and $S_{n}$ States

\begin{tabular}{|c|c|c|c|c|}
\hline \multirow[t]{2}{*}{ Excited states $^{a}$} & \multicolumn{2}{|c|}{$\operatorname{SOC}\left(\mathrm{cm}^{-1}\right)$} & \multirow[t]{2}{*}{ MO contribution $^{b}$} & \multirow[t]{2}{*}{ Excitation energy (in eV) } \\
\hline & $\left\langle\mathrm{S}_{1}\left|\mathrm{H}_{\mathrm{sO}}\right| \mathrm{T}_{\mathrm{n}}\right\rangle$ & $\left\langle\mathrm{S}_{2}\left|\mathrm{H}_{\mathrm{sO}}\right| \mathrm{T}_{\mathrm{n}}\right\rangle$ & & \\
\hline $\mathrm{S}_{1}$ & - & - & $H \rightarrow L(91 \%)$ & 2.25 \\
\hline $\mathrm{S}_{2}$ & - & - & $\mathrm{H}-1 \rightarrow \mathrm{L}(84 \%)$ & 2.60 \\
\hline $\mathrm{S}_{3}$ & - & - & $\mathrm{H}-2 \rightarrow \mathrm{L}(93 \%)$ & 2.80 \\
\hline $\mathrm{T}_{1}$ & 0.457 & 0.463 & $H \rightarrow L(96 \%)$ & 1.60 \\
\hline $\mathrm{T}_{2}$ & 0.154 & 0.494 & $\mathrm{H}-1 \rightarrow \mathrm{L}(87 \%)$ & 2.36 \\
\hline $\mathrm{T}_{3}$ & 1.964 & 0.676 & $\mathrm{H}-2 \rightarrow \mathrm{L}(82 \%)$ & 2.47 \\
\hline
\end{tabular}

${ }^{a}$ Only selected excited states were reported; ${ }^{b} \mathrm{H}$ and $\mathrm{L}$ stand for $\mathrm{HOMO}$ and LUMO, respectively. Only the main configurations are presented.

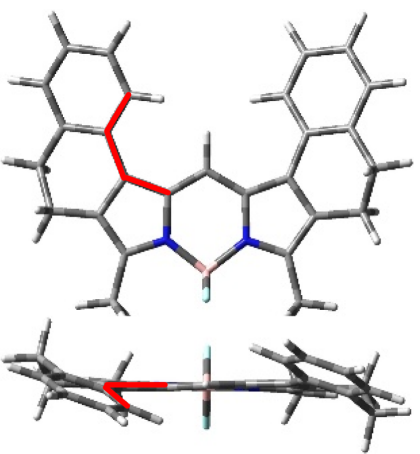

Fig. 6 Optimized ground state geometry (B3LYP/6-31G(d)) of BDP-1. The dihedral angles between Bodipy $\pi$-conjugation system and the peripheral phenyl rings are highlighted in red. The dihedral angles were determined as $21.4^{\circ}$ and $17.0^{\circ}$ by DFT calculation and single crystal $\mathrm{X}$-ray diffraction, respectively. ${ }^{46}$
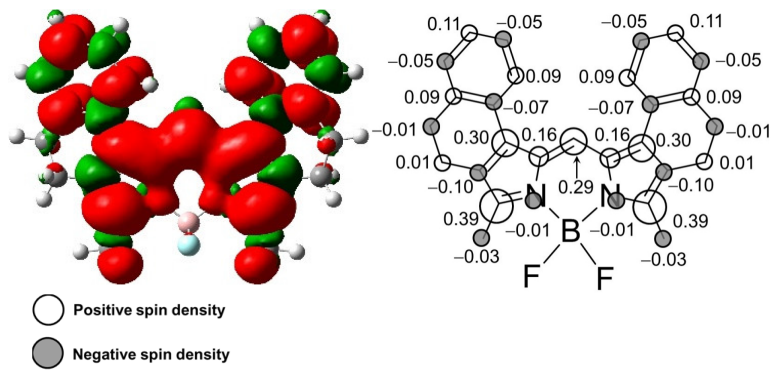

Fig. 7 Spin density isosurface of the lowest triplet state of BDP-1 at its optimized triple state geometry (Isovalue $=0.0004$ ). Calculated at the UB3LYP/6-31G(d) level.

indicating the geometries of excited state are slightly more planar than the ground state.

The triplet state spin density of BDP-1 was calculated based on optimized triplet state geometry (Fig. 7). The triplet state wave function distribution is delocalized on the overall $\pi$ conjugated system. This is in agreement with the small ZFS $D$ parameter observed with the TREPR spectra (Fig. 5).

To unravel the ISC of BDP-1 and to obtain accurate excitation energies, COSMO-RI-ADC2 $2^{67,68}$ single point calculations were performed (see Experimental Section). The ADC(2) method has been proved accurate for excited states of these systems. ${ }^{69}$ The calculation of the spin- orbit coupling matrix elements (SOCMEs) values was performed with TDDFT calculations (Table 3, Fig. 8 and details in the Experimental Section). The HOMO and LUMO are both mainly localized on Bodipy chromophore, not on the peripheral phenyl rings. However, the HOMO-2 and HOMO-1 are delocalized on the peripheral phenyl rings and the bridge- bearing ethylene group. The $\mathrm{S}_{1}$ state energy was determined as $2.25 \mathrm{eV}$, which is in agreement with the experimental data ( 2.20 $\mathrm{eV}$, the maximum absorption). In addition, the calculated energy for $\mathrm{S}_{2}$ and $\mathrm{S}_{3}$ states are $2.60 \mathrm{eV}$ and $2.80 \mathrm{eV}$, respectively, the transitions to these states contribute to the weak, broad absorption bands in the range of $400 \mathrm{~nm}$ to $500 \mathrm{~nm}(3.1 \sim 2.5 \mathrm{eV}$. Fig. 1). SOC values between the manifold of singlet and triplet states were calculated to determine the possible ISC channels (Table 3). For $S_{1} \rightarrow T_{n}$ state transitions, the SOC values between $\mathrm{S}_{1} \rightarrow \mathrm{T}_{1}$ is large enough for ISC $\left(0.457 \mathrm{~cm}^{-1}\right) \cdot{ }^{70,71}$ However, the $\mathrm{S}_{\mathrm{n}}-\mathrm{T}_{\mathrm{m}}$ energy gaps should be concomitantly analysed, as they also govern the efficiency of ISC. ${ }^{72}$ The energy gap between $\mathrm{S}_{1} / \mathrm{T}_{1}$ is too large for an efficient ISC $(0.65 \mathrm{eV})$. Therefore, the ISC process of $S_{1} \rightarrow T_{1}$ is unlikely. For other transitions, $S_{1} \rightarrow T_{2}$ and $\mathrm{S}_{1} \rightarrow \mathrm{T}_{3}$, the SOC values are non-zero $\left(0.154 \mathrm{~cm}^{-1}\right.$ and $\left.1.964 \mathrm{~cm}^{-1}\right)$ and the energy gaps are much smaller $(-0.11 \mathrm{eV}$ and $-0.22 \mathrm{eV}$, respectively). Therefore, $S_{1} \rightarrow T_{2} / T_{3}$ are responsible for the observed ISC in BDP-1. The $\mathrm{x}-$; $\mathrm{y}$-; and z-components of the

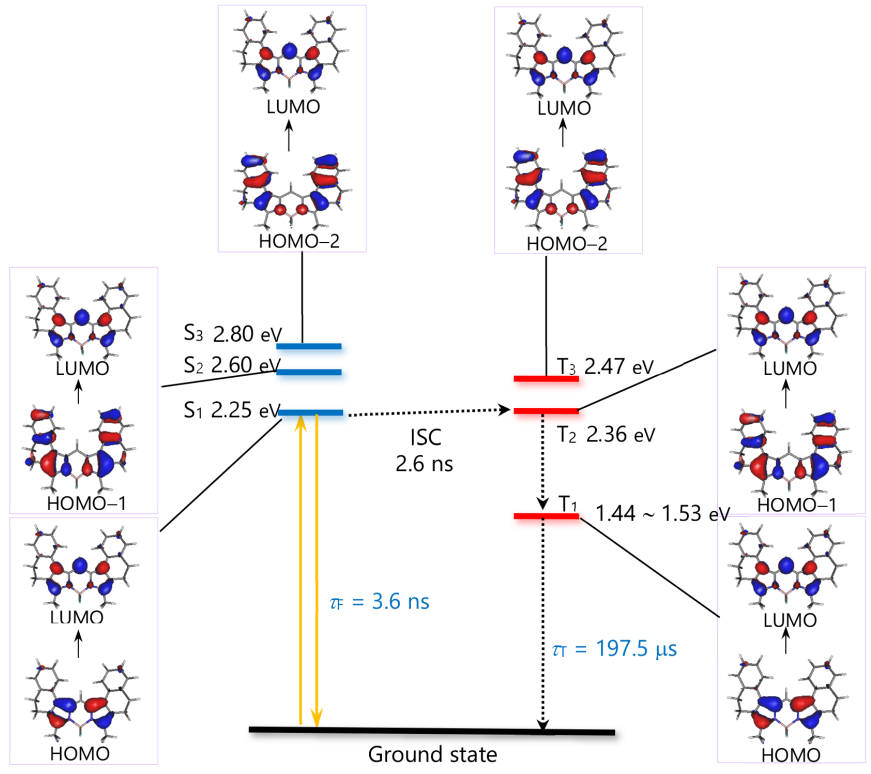

Fig. 8 Selected frontier molecular orbitals and photophysical process of BDP-1. IC and ISC stands for internal conversion and intersystem crossing, respectively. The energetic values are based on the COSMO-RI-ADC(2)/def2-TZVP calculations. Energy level of $T_{1}$ state was estimated by TTET method and energy levels of $T_{2}$ and $T_{3}$ state are determined by COSMO-RI-ADC(2)/def2-TZVP calculations. The $\tau_{\mathrm{T}}$ is the intrinsic lifetime of BDP-1 in solution obtained by fitting $\tau_{\top}$ in two different concentrations. ${ }^{47}$ 
computed SOCs values are listed in Table S2. As it can be seen in Table S2, and in agreement with the TREPR experiments, the $y$-component is the largest computed SOC component between the $S_{1}$ and $T_{2} / T_{3}$ excited states. This is in agreement with the overpopulation of the $T_{\mathrm{y}}$ substate measured for BDP-1 (Table 2). This piece of evidence puts on firmer grounds the assignments of the ISC processes.

The SOC value of $\mathrm{S}_{2} \rightarrow \mathrm{T}_{3}$ transition is also non-negligible large $\left(0.676 \mathrm{~cm}^{-1}\right)$ and the energy gap is also small $(0.13 \mathrm{eV})$. However, the time constant of ISC process by excitation to the $S_{2}$ state ( $\lambda_{\text {ex }}$ $=475 \mathrm{~nm}, 3.1 \mathrm{~ns})$ isn't larger than the value by excitation to the $\mathrm{S}_{1}$ state $\left(\lambda_{\text {ex }}=540 \mathrm{~nm}, 2.6 \mathrm{~ns}\right.$. Fig. 3 and Fig. S9†), indicating the ISC of $S_{2} \rightarrow T_{3}$, if there is at all, doesn't facilitate the ISC significantly. Therefore, we propose the $S_{1} \rightarrow T_{2} / T_{3}$ ISC channel to be the most relevant one for BDP-1.

The photophysical processes of BDP-1 are summarized in Fig. 8. $S_{1}$ state will be generated by photoexcitation of the molecule at $564 \mathrm{~nm}$. Afterward ISC process will occur between $\mathrm{S}_{1}$ and $T_{2} / T_{3}$ states, supported by calculated SOC values, the meantime of ISC is determined as 2.6 ns by femtosecond and subnanosecond transient absorption spectroscopy. Long triplet state lifetime was observed as $197.5 \mu$ s in fluid solution with nanosecond transient absorption spectroscopy. ${ }^{47}$ Exceptionally long-lived triplet state was obtained in solid polymer film at room temperature $(4.5 \mathrm{~ms})$.

\section{Application of BDP-1 in photodynamic therapy (PDT)}

The intermolecular triplet-triplet energy transfer, a typical primary photophysical process involved in the application of triplet PSs, such as the singlet oxygen $\left({ }^{1} \mathrm{O}_{2}\right)$ photosensitizing, will be enhanced by longer triplet state lifetime of PSs. Therefore, triplet PSs showing long-lived triplet state are superior to those triplet PSs showing shorter triplet state lifetimes. This postulation has been confirmed with phosphorescence oxygen sensing, ${ }^{73}$ and PDT studies (tumor tissue is normally in a hypoxia microenvironment). ${ }^{76} 79$

PDT is a promising method for cancer therapy, without the side effect of drug-resistance, and for its low dark toxicity and targeted treatment is feasible. Porphyrin derivatives were used as triplet PSs for PDT. ${ }^{80} 82$ Some new triplet PSs were reported recently, but the compounds usually contain heavy atoms to enhance the ISC. ${ }^{7,83}$ To overcome the disadvantage of the dark toxicity of the triplet PSs, heavy atom-free PDT reagents are highly desired. ${ }^{34,44,84}$ BDP-1 is one of such PDT reagents, due to its efficient single oxygen producing ability $\left(\Phi_{\Delta}=55.0 \%\right.$ in DCM) and long triplet state lifetime (197.5 $\mu \mathrm{s})$ in solution. ${ }^{47}$

In order to demonstrate the advantage of the long-lived triplet state of BDP-1 ( $\left.\tau_{\mathrm{T}}=197.5 \mu \mathrm{s}\right)$ for PDT study, ${ }^{47}$ the ${ }^{1} \mathrm{O}_{2}$ photosensitizing with the compounds as triplet PSs was studied under normal air atmosphere and severe hypoxia atmosphere $\left(0.2 \% \mathrm{O}_{2}\right.$ in $\left.\mathrm{N}_{2}, \mathrm{v} / \mathrm{v}\right)$, and the results are compared to the Bodipy analogue triplet PS (BDP-3) but showing much shorter lived triplet state $\left(\tau_{T}=1.8 \mu \mathrm{s}\right.$. Fig. 9$) .^{49}$ Similar consumption rate of 1,3-diphenylisobenzofuran (DPBF. ${ }^{1} \mathrm{O}_{2}$ scavenger) was observed under sever hypoxia and air atmosphere for BDP-1. The $\Phi_{\Delta}$ is $50.0 \%$ under hypoxia atmosphere, which is slightly lower than that under normal air atmosphere (Fig. 9a. $\Phi_{\Delta}=55.0 \%$ ). The
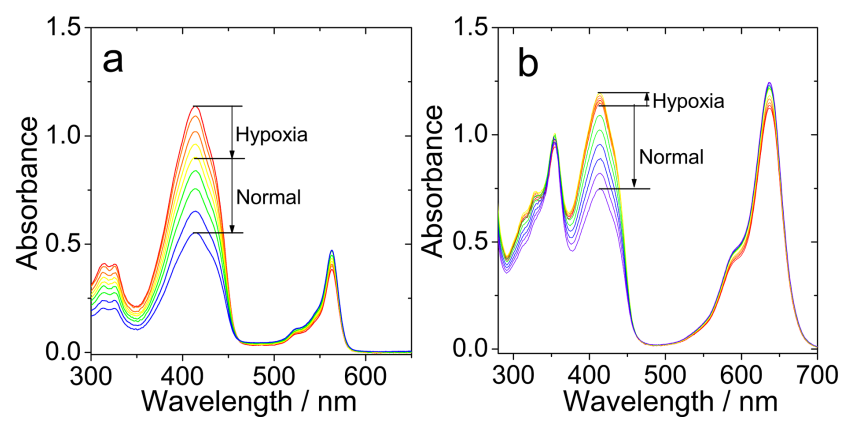

Fig. 9 Monitoring the ${ }^{1} \mathrm{O}_{2}$ production using UV-Vis absorption changes of the DPBF upon photo-irradiation with (a) BDP-1 (b) BDP-3 as triplet PSs under severe hypoxia atmosphere $\left(0.2 \% \mathrm{O}_{2}\right.$ in $\left.\mathrm{N}_{2}, \mathrm{v} / \mathrm{v}\right)$ and normal atmosphere (air condition, $\left.21 \% \mathrm{O}_{2}\right)$ in $\mathrm{DCM}$. Note: the increase of absorbance of DPBF in hypoxia atmosphere in (b) is mainly due to the evaporation of DCM. Optically matched solution was used, $\lambda_{\mathrm{ex}}=570 \mathrm{~nm}$, the irradiation time for each data point is $20 \mathrm{~s}\left(6 \mathrm{~mJ} \mathrm{~cm}^{2}\right) .20^{\circ} \mathrm{C}$.
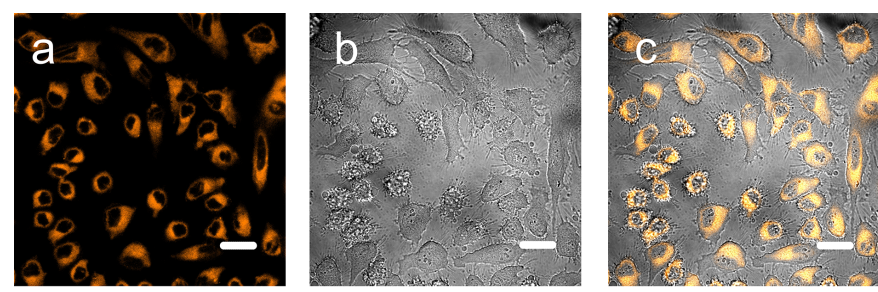

Fig. 10 Confocal microscopy imaging of HeLa cells after incubation with $5 \mu$ M BDP-1. (a) Fluorescence channel (570-700 nm); (b) Bright channel; (c) Merged image. $\lambda_{\text {ex }}=559 \mathrm{~nm}$, $20^{\circ} \mathrm{C}$, scale bar: $20 \mu \mathrm{m}$.

environment, which is ideal for PDT. Therefore, BDP-1 was used as a novel reagent for PDT.

HeLa cell were incubated with BDP-1 $(5 \mu \mathrm{M})$ for $24 \mathrm{~h}$ and the cellular imaging was studied by confocal laser scanning microscopy (Fig. 10). Orange fluorescence was observed clearly in cell cytoplasm with excitation of $559 \mathrm{~nm}$ laser, which indicate compound BDP-1 shows efficient cellular uptake.

The phototoxicity and dark toxicity can be evaluated by using 3-(4,5-dimethyl-2-thiazolyl)-2,5-diphenyl tetrazolium bromide (MTT) assays (Fig. 11). Hela cell were incubated with different doses of BDP-1 for $24 \mathrm{~h}$. Then the experimental group was photoirradiated $\left(560 \mathrm{~nm}, 3.6 \mathrm{~J} \mathrm{~cm}^{-}{ }^{2}\right.$ ) and the control group was incubated in dark environment. As shown in Fig. 11, upon result indicates that BDP-1 is efficient even in a hypoxia environment. On the contrary, compound BDP-3 shows negligible ${ }^{1} \mathrm{O}_{2}$ production under severe hypoxia condition $\left(\Phi_{\Delta} \approx 0 \%\right)$, although the ${ }^{1} \mathrm{O}_{2}$ production is efficient under normal air atmosphere (Fig. 9b. $\Phi_{\Delta}=54.5 \%$ ). Our results show that the BDP-1, without the detrimental HAE to shorten the triplet state lifetime, is a more potent ${ }^{1} \mathrm{O}_{2}$ producing reagent in hypoxia photoirradiation, the cell viability was decreased significantly, indicating high phototoxicity of BDP-1 $\left(\mathrm{EC}_{50}=1.0 \mu \mathrm{M}\right)$. The cell viability of control group without irradiation shows no obvious decrease. Thus BDP-1 shows good biocompatibility (low dark toxicity).

This light toxicity $\left(\mathrm{EC}_{50}=1.0 \mu \mathrm{M}\right)$ is comparable to that of the Bodipy photosensitizers based on HAE $(0.2 \sim 10 \mu \mathrm{M}))^{7,85,86}$ Moreover, the dark toxicity of BDP-1 is much lower $\left(\mathrm{EC}_{50}=78.5\right.$ $\mu \mathrm{m})$ (Fig. S14 $\dagger$ ) than BDP-2 PDT reagent $\left(\mathrm{EC}_{50}=6.0 \mu \mathrm{m}\right){ }^{78}$ demonstrating the advantage of heavy atom-free PDT reagent. 


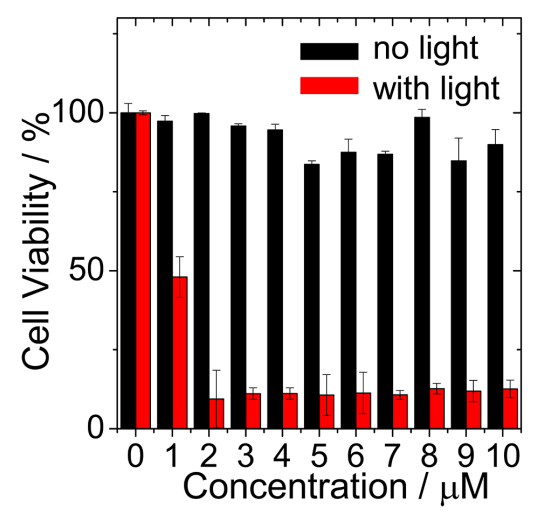

Fig. 11 Comparison of the cell viability of HeLa cells with pre-treated BDP-1 with different concentration, with and without light irradiation (560 nm LED, $\left.3.6 \mathrm{~J} \mathrm{~cm}{ }^{2}\right), 20^{\circ} \mathrm{C}$.

$0 \mathrm{~s}$
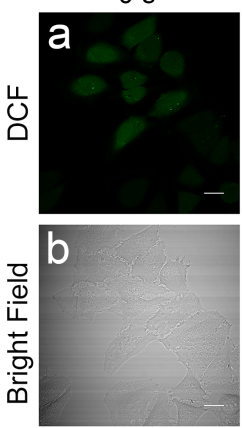

d
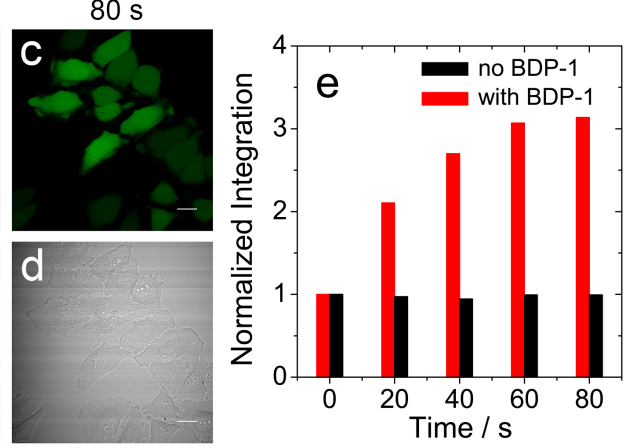

Fig. 12 Detection of intracellular ROS generation in HeLa cells. The cells were irradiation with $559 \mathrm{~nm}$ after incubated with $3 \mu \mathrm{M}$ BDP-1 and DCF-DA. (a) and (c) the channel of fluorescence image of DCF $\left(\lambda_{\mathrm{ex}}=488 \mathrm{~nm} ; \lambda_{\mathrm{em}}=500-540 \mathrm{~nm}\right)$; (b) and (d) transmitted light images. Scale bar: $20 \mu \mathrm{m}$. (e) The normalized integration of fluorescence image upon different irradiation time in presence and absence of BDP-1. Note: the intensity integration with $0 \mathrm{~s}$ irradiation was normalized as 1.

To confirm the cell death is triggered by intracellular ROS generation, 2',7'-dichlorodihydrofluorescein diacetate (DCFDA) was used as the probe of ROS generated by BDP-1 upon photo-irradiation (Fig. 12). The molecular probe will be deacetylated by ROS and the product is green fluorescent. The HeLa cells was pre-treated by DCF-DA and BDP-1, following the irradiation of $559 \mathrm{~nm}$, which give rise to obvious enhancement of green fluorescence (Fig. 12c and Fig. S15 $)$ ). As a control, negligible increase of green fluorescence was observed after irradiation for the DCF-DA pre-treated HeLa cells in absence of BDP-1 (Fig. S16 $†$ ). The comparison of fluorescence integration upon different irradiation time also demonstrate that the unambiguous enhancement of green fluorescence produced by intracellular ${ }^{1} \mathrm{O}_{2}$ in presence of both BDP-1 and DCF-DA (Fig. 12e). It is noteworthy that megascopic formation of membrane blebs was observed accompanying the ${ }^{1} \mathrm{O}_{2}$ generation, indicating the cells are undergoing death in the presence of BDP-1 and photo-irradiation.

\section{Conclusions}

In summary, detailed photophysical properties of heavy atomfree photosensitizer Bodipy derivative with twisted $\pi$ conjugation framework were studied. We found efficient intersystem crossing (ISC) for Bodipy derivative with twisted $\pi$ conjugation framework. Femtosecond and sub-nanosecond transient absorption spectral studies show the ISC time constant is 2.6 ns. Theoretical computations show the spinorbital coupling matrix elements (SOCME) are $0.154 \mathrm{~cm}^{-1}$ for $\mathrm{S}_{1} / \mathrm{T}_{2}$ states and $1.964 \mathrm{~cm}^{-1}$ for $\mathrm{S}_{1} / \mathrm{T}_{3}$ states, and the existence of matched $\mathrm{S}_{1} / \mathrm{T}_{2}$ or $\mathrm{S}_{1} / \mathrm{T}_{3}$ energy (energy gap: $0.11 \mathrm{eV}$ and $0.22 \mathrm{eV}$, respectively). In comparison, the unsubstituted Bodipy with planar $\pi$-conjugation framework shows SOC $\left(0.19 \mathrm{~cm}^{-1}\right.$ for $S_{1} / T_{1}$ states) but the much larger energy gap $(0.69 \mathrm{eV})$ inhibits the ISC. Time-resolved electron paramagnetic resonance (TREPR) spectroscopy confirmed the delocalization of the triplet state wave function on the whole molecule, the zero-field splitting (ZFS) parameter is $D=-69.5 \mathrm{mT}$, which is smaller than the 2,6diiodoBodipy $(D=-104.6 \mathrm{mT})$. The electron spin selectivity of the twisted Bodipy (population ratio of the sublevels of the triplet state, $P_{\mathrm{x}}: P_{\mathrm{y}}: P_{\mathrm{z}}=0.00: 1.00: 0.21$ ) is also different from that accessed with the normal spin-orbit coupling, enhanced by heavy atom effect (for the triplet state of 2,6-diiodoBodipy, $P_{\mathrm{x}}$ : $\left.P_{\mathrm{y}}: P_{\mathrm{z}}=0.20: 0.00: 1.00\right)$. The advantage of using the twisted $\pi-$ conjugation framework to enhance the ISC, instead of the typical heavy atom effect to enhance the ISC, is the long-lived triplet state (intrinsic triplet state lifetime $\tau_{\mathrm{T}}=197.5 \mu \mathrm{s}$ in solution, exceptionally long triplet state lifetime in solid polymer film, $\tau_{T}=4.5 \mathrm{~ms}$ ). The advantage of the longer triplet state lifetime has been demonstrated by the efficient singlet oxygen photosensitizing even under severe hypoxia atmosphere $\left(\mathrm{pO}_{2}=0.2 \%\right.$ in $\left.\mathrm{N}_{2}, \mathrm{v} / \mathrm{v}\right)$. In comparison the triplet photosensitizer 2,6-diiodobisstyrylbodipy showing short triplet state lifetime $(1.8 \mu \mathrm{s})$ gives negligible ${ }^{1} \mathrm{O}_{2}$ photosensitizing ability in the hypoxia environment. The application of the twisted Bodipy as novel triplet PS was demonstrated with the cellular photodynamic therapy study, high phototoxicity $\left(\mathrm{EC}_{50}=\right.$ $1.0 \mu \mathrm{M})$ and low dark toxicity $\left(\mathrm{EC}_{50}=78.5 \mu \mathrm{M}\right)$ was observed with HeLa cells. Our results indicate that the chromophores with twisted $\pi$-conjugation structure may become a novel type of potent triplet PS.

\section{Experimental Section}

\section{Materials and equipment}

All chemicals used in experiment are analytically pure. Solvents for synthesis were freshly dried over suitable drying agents before using. ${ }^{1} \mathrm{H}$ NMR spectra were recorded on a $400 \mathrm{MHz}$ Bruker NMR spectrometer. High resolution mass spectrum (HRMS) were recorded by ESI-HRMS spectrometer. Fluorescence spectra were recorded on FS5 fluorescence spectrophotometer (Edinburgh Instrument Ltd, UK). UV-Vis absorption spectra were recorded on HP8453A UV-Vis spectrophotometer (Agilent Ltd, USA). Time resolved fluorescence spectra were recorded on OB920 luminescence lifetime spectrometer, equipped with picosecond EPL picosecond pulsed diode laser (Edinburgh Instrument Ltd, UK). 
The absolute fluorescence quantum yields were determined by C13534 UV-NIR absolute photoluminescence quantum yield spectrometer, equipped with optical integration sphere (Hamamatsu Photonics Ltd., Japan).

\section{Nanosecond transient absorption spectra}

Nanosecond transient absorption spectra were recorded on LP980 laser flash-photolysis spectrometer (Edinburgh Instrument Ltd, UK), equipped with OPO laser (Opolette ${ }^{\mathrm{TM}}$, the wavelength is tunable in the range of $210-2400 \mathrm{~nm}$. OPOTEK, USA), typical laser power is ca. $5 \mathrm{~mJ}$ per pulse. The data (kinetic decay curve and spectrum) were obtained with the L900 software. All samples were deaerated with $\mathrm{N}_{2}$ for ca. $15 \mathrm{~min}$ before measurement.

\section{Triplet state quantum yield $\left(\Phi_{T}\right)$}

The triplet state quantum yields were determined by ground state bleaching method according to the equation 2 . The data were measured by nanosecond transient absorption spectroscopy.

$$
\Phi_{\text {sam }}=\Phi_{\text {std }}\left(\frac{\varepsilon_{\text {std }}}{\varepsilon_{\text {sam }}}\right)\left(\frac{\Delta \mathrm{A}_{\text {sam }}}{\Delta \mathrm{A}_{\text {std }}}\right)
$$

In the eq. 2, "sam" and "std" represent sample and standard, respectively. $\Phi$ is the triplet state quantum yield, $\varepsilon$ is the molar absorption coefficient, which is determined by UV-Vis absorption spectra, $\Delta A$ is the optical intensity of the ground state bleaching band determined by nanosecond transient absorption spectroscopy. Optically matched solutions were used for the measurement of determination of $\Delta A$.

\section{Femtosecond transient absorption spectroscopy}

The fs-TA experiments were performed using a Ti:sapphire laser system with $\sim 100 \mathrm{fs}$ pulse duration and $1 \mathrm{kHz}$ repetition rate (Astrella, Coherent)) and a commercial setup of an ultrafast transient absorption spectrometer (Ultrafast System, Helios). The excitation wavelength was determined from the steady UVVis absorption spectra and generated in an optical parametric amplifier (TOPAS, Light Conversion. Magic angle between the probe and the pump beam polarization direction was used. The Surface Xplorer and Glotaran software were used for processing the experimental data basing on data after chirp correction. ${ }^{87}$

\section{Sub-nanosecond transient absorption spectroscopy}

The sub-ns TA experiment was performed using an ultrafast Systems EOS UV-NIR spectrometer with broadband capability. In EOS experiment, the probe pulse was a white-light continuum generated by a super continuum source. The ultrafast pump pulses were generated in an optical parametric amplifier (TOPAS, Light Conversion). The Surface Xplorer and Glotaran software were used for processing the experimental data basing on data after chirp correction. ${ }^{87}$

Time resolved electron paramagnetic resonance (TREPR) spectroscopy
TREPR measurements were performed with a continuous wave/TREPR setup based on an X-band Bruker EMX spectrometer $(9.7 \mathrm{GHz})$ equipped with a $\mathrm{N}_{2}$-cooled temperature control system $(T \approx 80-300 \mathrm{~K})$. In all cases the samples were shock-frozen in liquid nitrogen, then the samples were transferred into the probe and the temperature was equilibrated for several minutes. A $532 \mathrm{~nm} \mathrm{Nd:YaG} \mathrm{laser} \mathrm{LOTIS-}$ TII was used as excitation power source. The samples' concentrations were set to have the full light absorption at the width of the quartz EPR tube $(2.8 \mathrm{~mm})$. In all cases, solution was placed into the quartz EPR tube, evacuated $\left(10^{2}\right.$ Torr pressure), and sealed off. EasySpin was used for simulations of the experimental TREPR spectra. ${ }^{88}$

\section{Quantum chemical investigations}

All optimizations are based on density functional theory (DFT) or TD-DFT calculations. The B3LYP functional was used along with the $6-31 G^{*}$ basis set. The ground state $\left(S_{0}\right)$ and excited state $\left(S_{1}, T_{1}\right)$ geometries of BDP-1 were optimized with DFT and TD-DFT, respectively (without symmetry constraints); using Gaussian 09W and Gausssian16. ${ }^{89,90}$ To obtain accurate excitation energies, single point calculations using the algebraic diagrammatic construction method of second order, ${ }^{67}$ i.e., ADC (2), were performed. The latter calculations were performed in combination with the def2-TZVP basis set. Solvent effects were considered with the Conductor-like screening model (COSMO) for the latter calculations using dichloromethane (permittivity, $\varepsilon=8.930$ ) as a solvent. COSMO-RI-ADC(2)/def2-TZVP ${ }^{68}$ calculations were done using Turbomole 7.1. ${ }^{91}$ For the calculation of spin-orbit couplings (SOCs) matrix elements we have performed TDDFT calculations in Orca $4.2^{92.94}$ The latter calculations were performed using the B3LYP functional. Relativistic effects were considered by using the zero-order relativistic approximation (ZORA). The ZORA-def2-TZVP basis sets ${ }^{95}$ were used for all the atoms and solvent effects in DCM were considered with the COSMO model. The spin-orbit integrals were calculated using the one-electron mean-field approximation which included resolution of identity approximation (RI), i.e., the $\mathrm{RI}-\mathrm{SOMF}(1 \mathrm{X})^{96}$ approximation. These calculations were performed at the $S_{1}$ optimized geometry.

\section{Photodynamic Therapy}

The HeLa cells were cultured in Dulbecco's modified Eagle's medium (DMEM) for $24 \mathrm{~h}$ incubation $\left(37^{\circ} \mathrm{C}, 5 \% \mathrm{CO}_{2}\right)$. The BDP$1(5 \mu \mathrm{M})$ was added and the cells were incubated for $24 \mathrm{~h}$ in dark. Before the cells were imaged by laser scanning confocal microscope, the cells were washed with PBS buffer solution for three times to remove the remnant dyad. The cytotoxicity test were assessed by 3-(4,5-dimethyl-2-thiazolyl)-2,5-diphenyl tetrazolium bromide (MTT) assays. After incubation in DMEM for $24 \mathrm{~h}$ incubation $\left(37^{\circ} \mathrm{C}, 5 \% \mathrm{CO}_{2}\right)$, Hela cells were incubated with different doses of dyad. After $24 \mathrm{~h}$ incubation in dark, the experimental group was exposure to yellow LED light (560 nm, $1 \mathrm{~mW} \mathrm{~cm}^{2}$ ) for $1 \mathrm{~h}$ and the control group was incubated in dark environment. The cells were further cultured for $4 \mathrm{~h}$ and $10 \mu \mathrm{l}$ freshly prepared MTT ( $5 \mathrm{mg} \mathrm{mL}^{-1}$ ) solution was added into each 
well. The supernatant liquid in each well was removed after $4 \mathrm{~h}$ incubation and $100 \mu \mathrm{l}$ DMSO was added to dissolve the formed formazan solid. Finally, the absorbance at $490 \mathrm{~nm}$ was measured with a Bio-Rad microplate reader.

\section{Author contributions}

J. Z. is responsible for the conceptualization of the manuscript and designed the project; Y.D. is responsible for part of the data curation, spectral measurements and data analysis; D. E. and P. $K$. performed the quantum chemical investigations and data analysis; P. M. and O. F. M. performed the femtosecond transient absorption and sub-nanosecond transient absorption measurements and the data analysis; I. K. and M. F. performed TREPR spectral studies and data analysis; S. L. and H. W. did photodynamic therapy study; A. E. and A. K. did analysis of part of the femtosecond transient absorption data; J. Z., D. E., W. H., A. K., O. F. M. and M. F. wrote the manuscript. All the authors discussed extensively the results presented and reviewed the final version of the manuscript.

\section{Conflicts of interest}

There are no conflicts to declare.

\section{Acknowledgements}

J. Z. thanks the NSFC (U2001222, 21673031, 21761142005 and 21911530095) and the State Key Laboratory of Fine Chemicals (ZYTS201901) for financial support. M.F. thanks the Ministry of High Education and Science of Russia for access to EPR equipment. I.K. thanks RFBR (grant no. 19-29-10035) for financial support. D. E. thanks funding from KU Leuven Internal Funds.

\section{Notes and references}

1 J. Xuan and W.-J. Xiao, Angew. Chem. Int. Ed., 2012, 51, 6828-6838.

2 L. Shi and W. Xia, Chem. Soc. Rev., 2012, 41, 7687-7697.

3 D. Ravelli, M. Fagnoni and A. Albini, Chem. Soc. Rev., 2013, 42, 97-113.

4 T. N. Singh-Rachford and F. N. Castellano, Coordin. Chem. Rev., 2010, 254, 2560-2573.

5 J. Zhou, Q. Liu, W. Feng, Y. Sun and F. Li, Chem. Rev., 2015, 115, 395-465.

6 N. Yanai and N. Kimizuka, Accounts. Chem. Res., 2017, 50, 2487-2495.

7 A. Kamkaew, S. H. Lim, H. B. Lee, L. V. Kiew, L. Y. Chung and K. Burgess, Chem. Soc. Rev., 2013, 42, 77-88.

8 O. J. Stacey and S. J. A. Pope, RSC Adv., 2013, 3, 25550-25564.

9 J. Tian, L. Ding, H.-J. Xu, Z. Shen, H. Ju, L. Jia, L. Bao and J.-S. Yu, J. Am. Chem. Soc., 2013, 135, 18850-18858.

10 X.-J. Jiang, J. T. F. Lau, Q. Wang, D. K. P. Ng and P.-C. Lo, Chem. Eur. J., 2016, 22, 8273-8281.

11 J. Liu, C. Jin, B. Yuan, X. Liu, Y. Chen, L. Ji and H. Chao, Chem. Commun., 2017, 53, 2052-2055.

12 (a) Z. Yan, M. Wang, M. Shi, Y. He, Y. Zhang, S. Qiu, H. Yang, H. Chen, H. He and Z. Guo, J. Mater. Chem. B, 2020, 8, 6886-6897. (b) V.-N. Nguyen, S. J. Park, S. Qi, J. Ha, S. Heo, Y. Yim, G. Baek, C. S. Lim, D. J. Lee, H. M. Kim, J. Yoon. Chem. Commun., 2020, 56, 11489-11492. (c) V.-N. Nguyen, S. Qi, S. Kim, N. Kwon, G. Kim, Y. Yim, S. Park, and J. Yoon. J. Am. Chem. Soc., 2019, 141, 16243-16248.

13 R. Prieto-Montero, R. Sola-Llano, R. Montero, A. Longarte, T. Arbeloa, I. López-Arbeloa, V. Martínez-Martínez and S. Lacombe, Phys. Chem. Chem. Phys., 2019, 21, 20403-20414.

14 (a) N. Epelde-Elezcano, E. Palao, H. Manzano, A. PrietoCastañeda, A. R. Agarrabeitia, A. Tabero, A. Villanueva, S. de la Moya, Í. López-Arbeloa, V. Martínez-Martínez and M. J. Ortiz, Chem. Eur. J., 2017, 23, 4837-4848. (b) Z. Ruan, W. Miao, P. Yuan, L. Le, L. Jiao, E. Hao, and L. Yan. Bioconjugate Chem., 2018, 29, 3441-3451

15 J. Zhao, W. Wu, J. Sun and S. Guo, Chem. Soc. Rev., 2013, 42, 5323-5351.

16 Y.-L. Chen, S.-W. Li, Y. Chi, Y.-M. Cheng, S.-C. Pu, Y.-S. Yeh and P.T. Chou, Chemphyschem, 2005, 6, 2012-2017.

17 N. J. Turro, V. Ramamurthy and J. C. Scaiano, Principles of molecular photochemistry: an introduction, University science books, 2009.

18 R. R. Islangulov, D. V. Kozlov and F. N. Castellano, Chem. Commun., 2005, 3776-3778.

19 T. Yogo, Y. Urano, Y. Ishitsuka, F. Maniwa and T. Nagano, J. Am. Chem. Soc., 2005, 127, 12162-12163.

20 C. A. Parker and T. A. Joyce, Chem. Commun. (London), 1968, 749-750.

21 T. N. Singh-Rachford and F. N. Castellano, J. Phys. Chem. A, 2009, 113, 5912-5917.

22 T. J. Penfold, E. Gindensperger, C. Daniel and C. M. Marian, Chem. Rev., 2018, 118, 6975-7025.

23 W. Wu, J. Zhao, J. Sun and S. Guo, J. Org. Chem., 2012, 77, 5305-5312.

24 Y. Liu and J. Zhao, Chem. Commun., 2012, 48, 3751-3753.

25 P. Yang, W. Wu, J. Zhao, D. Huang and X. Yi, J. Mater. Chem., 2012, 22, 20273-20283.

26 L. Huang, X. Yu, W. Wu and J. Zhao, Org. Lett., 2012, 14, 2594-2597.

27 A. Kawai and K. Obi, J. Phys. Chem., 1992, 96, 52-56.

28 Z. Wang, J. Zhao, A. Barbon, A. Toffoletti, Y. Liu, Y. An, L. Xu, A. Karatay, H. G. Yaglioglu, E. A. Yildiz and M. Hayvali, J. Am. Chem. Soc., 2017, 139, 7831-7842.

29 M. Bröring, R. Krüger, S. Link, C. Kleeberg, S. Köhler, X. Xie, B. Ventura and L. Flamigni, Chem. Eur. J., 2008, 14, 2976-2983.

30 B. Ventura, G. Marconi, M. Bröring, R. Krüger and L. Flamigni, New J. Chem., 2009, 33, 428-438.

31 D. Casanova, Chem. Rev., 2018, 118, 7164-7207.

32 R. Ziessel, B. D. Allen, D. B. Rewinska and A. Harriman, Chem. Eur. J., 2009, 15, 7382-7393.

33 J. W. Verhoeven, J. Photochem. Photobiol., C, 2006, 7, 40-60.

34 M. A. Filatov, S. Karuthedath, P. M. Polestshuk, H. Savoie, K. J. Flanagan, C. Sy, E. Sitte, M. Telitchko, F. Laquai, R. W. Boyle and M. O. Senge, J. Am. Chem. Soc., 2017, 139, 6282-6285.

35 Z. Yu, Y. Wu, Q. Peng, C. Sun, J. Chen, J. Yao and H. Fu, Chem. Eur. J., 2016, 22, 4717-4722.

36 M. Sapir and E. V. Donckt, Chem. Phys. Lett., 1975, 36, 108-110.

37 K. Schmidt, S. Brovelli, V. Coropceanu, D. Beljonne, J. Cornil, C. Bazzini, T. Caronna, R. Tubino, F. Meinardi, Z. Shuai and J.-L. Brédas, J. Phys. Chem. A, 2007, 111, 10490-10499.

38 S. Menning, M. Krämer, A. Duckworth, F. Rominger, A. Beeby, A. Dreuw and U. H. F. Bunz, J. Org. Chem., 2014, 79, 6571-6578.

39 Y. Hong, J. Oh, Y. M. Sung, Y. Tanaka, A. Osuka and D. Kim, Angew. Chem. Int. Ed., 2017, 56, 2932-2936.

40 F. Ema, M. Tanabe, S. Saito, T. Yoneda, K. Sugisaki, T. Tachikawa, S. Akimoto, S. Yamauchi, K. Sato, A. Osuka, T. Takui and Y. Kobori, J. Phys. Chem. Lett., 2018, 9, 2685-2690.

41 Y. Wu, Y. Zhen, Y. Ma, R. Zheng, Z. Wang and H. Fu, J. Phys. Chem. Lett., 2010, 1, 2499-2502. 
42 K. Nagarajan, A. R. Mallia, K. Muraleedharan and M. Hariharan, Chem. Sci., 2017, 8, 1776-1782.

43 H. Ito, H. Sakai, Y. Suzuki, J. Kawamata and T. Hasobe, Chem. Eur. J., 2020, 26, 316-325.

44 Z. Wang, L. Huang, Y. Yan, A. M. El-Zohry, A. Toffoletti, J. Zhao, A. Barbon, B. Dick, O. F. Mohammed and G. Han, Angew. Chem. Int Ed., 2020, 59, 16114-16121.

45 K. Nagarajan, A. R. Mallia, V. S. Reddy and M. Hariharan, J. Phys. Chem. C, 2016, 120, 8443-8450.

46 Y.-W. Wang, A. B. Descalzo, Z. Shen, X.-Z. You and K. Rurack Chem. Eur. J., 2010, 16, 2887-2903.

47 Y. Dong, B. Dick and J. Zhao, Org. Lett., 2020, 22, 5535-5539.

48 J.-H. Kim, F. Deng, F. N. Castellano and J.-H. Kim, Chem. Mater., 2012, 24, 2250-2252.

49 J. Ma, X. Yuan, B. Küçüköz, S. Li, C. Zhang, P. Majumdar, A. Karatay, X. Li, H. Gul Yaglioglu, A. Elmali, J. Zhao and M. Hayvali, J. Mater. Chem. C, 2014, 2, 3900-3913.

50 W. Wu, X. Cui and J. Zhao, Chem. Commun., 2013, 49, 9009-9011.

51 D. Lee, O. Bolton, B. C. Kim, J. H. Youk, S. Takayama and J. Kim, J. Am. Chem. Soc., 2013, 135, 6325-6329.

52 X. Ma, J. Wang and H. Tian, Accounts. Chem. Res., 2019, 52, $738-748$.

53 T. Zhang, X. Ma and H. Tian, Chem. Sci., 2020, 11, 482-487.

54 Z. Lou, Y. Hou, K. Chen, J. Zhao, S. Ji, F. Zhong, Y. Dede and B. Dick, J. Phys. Chem. C, 2018, 122, 185-193.

55 S. Richert, C. E. Tait and C. R. Timmel, J. Magn. Reson., 2017, 280, 103-116.

56 J. Telser, in eMagRes, 2017, 6, 207-234.

57 S. Weber, in eMagRes 2017, 6, 255-270.

58 H. Levanon and J. R. Norris, Chem. Rev., 1978, 78, 185-198.

59 H. van Willigen, G. Jones and M. S. Farahat, J. Phys. Chem., 1996, 100, 3312-3316.

60 A. Kawai and K. Shibuya, J. Photochem. Photobiol., C, 2006, 7, 89-103.

61 Y. E. Kandrashkin, Appl. Magn. Reson., 2019, 50, 1067-1082.

62 Y. E. Kandrashkin and A. v. d. Est, J. Chem. Phys., 2019, 151, 184301.

63 Y. Hou, T. Biskup, S. Rein, Z. Wang, L. Bussotti, N. Russo, P. Foggi, J. Zhao, M. Di Donato, G. Mazzone and S. Weber, J. Phys. Chem. C, 2018, 122, 27850-27865.

64 Z. Wang, A. A. Sukhanov, A. Toffoletti, F. Sadiq, J. Zhao, A. Barbon, V. K. Voronkova and B. Dick, J. Phys. Chem. C, 2019, 123 265-274.

65 Y. E. Kandrashkin, Z. Wang, A. A. Sukhanov, Y. Hou, X. Zhang, Y. Liu, V. K. Voronkova and J. Zhao, J. Phys. Chem. Lett., 2019, 10, 4157-4163.

66 Y. Hou, I. Kurganskii, A. Elmali, H. Zhang, Y. Gao, L. Lv, J. Zhao, A. Karatay, L. Luo and M. Fedin, J. Chem. Phys., 2020, 152, 114701.

67 A. Dreuw and M. Wormit, Wiley Interdiscip. Rev.: Comput. Mol. Sci., 2015, 5, 82-95.

68 S. Karbalaei Khani, A. Marefat Khah and C. Hättig, Phys. Chem. Chem. Phys., 2018, 20, 16354-16363.

69 J. A. González-Vera, F. Lv, D. Escudero, A. Orte, X. Guo, E. Hao, E. M. Talavera-Rodriguez, L. Jiao, N. Boens and M. J. Ruedas-Rama, Dyes. Pigments, 2020, 182, 108510.

70 S. Ji, J. Ge, D. Escudero, Z. Wang, J. Zhao and D. Jacquemin, J. Org. Chem., 2015, 80, 5958-5963.

71 G. Baryshnikov, B. Minaev and H. Ågren, Chem. Rev., 2017, 117, 6500-6537.

72 C. M. Marian, Wiley Interdiscip. Rev.: Comput. Mol. Sci., 2012, 2, 187-203.

73 S. Ji, W. Wu, W. Wu, P. Song, K. Han, Z. Wang, S. Liu, H. Guo and J. Zhao, J. Mater. Chem., 2010, 20, 1953-1963.

74 H. Guo, S. Ji, W. Wu, W. Wu, J. Shao and J. Zhao, Analyst, 2010, 135, 2832-2840.

75 W. Wu, W. Wu, S. Ji, H. Guo and J. Zhao, Dalton Trans., 2011, 40, 5953-5963.
76 R. Lincoln, L. Kohler, S. Monro, H. Yin, M. Stephenson, R. Zong, A. Chouai, C. Dorsey, R. Hennigar, R. P. Thummel and S. A. McFarland, J. Am. Chem. Soc., 2013, 135, 17161-17175.

77 X. Li, S. Kolemen, J. Yoon and E. U. Akkaya, Adv. Funct. Mater., 2017, 27, 1604053.

78 Z. Wang, M. Ivanov, Y. Gao, L. Bussotti, P. Foggi, H. Zhang, N. Russo, B. Dick, J. Zhao, M. Di Donato, G. Mazzone, L. Luo and M. Fedin, Chem. Eur. J., 2020, 26, 1091-1102.

79 Y. Hou, Q. Liu and J. Zhao, Chem. Commun., 2020, 56, 1721-1724.

80 R. Bonnett, Chem. Soc. Rev., 1995, 24, 19-33.

81 A. B. Ormond and H. S. Freeman, Materials, 2013, 6, 817-840.

82 A. S. Lavado, V. M. Chauhan, A. Alhaj Zen, F. Giuntini, D. R. E. Jones, R. W. Boyle, A. Beeby, W. C. Chan and J. W. Aylott, Nanoscale, 2015, 7, 14525-14531.

83 W. Sun, X. Zhao, J. Fan, J. Du and X. Peng, Small, 2019, 15, 1804927.

84 Y. Cakmak, S. Kolemen, S. Duman, Y. Dede, Y. Dolen, B. Kilic, Z. Kostereli, L. T. Yildirim, A. L. Dogan, D. Guc and E. U. Akkaya, Angew. Chem. Int. Ed., 2011, 50, 11937-11941.

85 P. Majumdar, X. Yuan, S. Li, B. Le Guennic, J. Ma, C. Zhang, D. Jacquemin and J. Zhao, J. Mater. Chem. B, 2014, 2, 2838-2854.

86 C. S. Kue, S. Y. Ng, S. H. Voon, A. Kamkaew, L. Y. Chung, L. V. Kiew and H. B. Lee, Photochem. Photobiol. Sci., 2018, 17, 1691-1708.

87 J. Snellenburg, J., S. Laptenok, R. Seger, K. Mullen, M. and I. Van Stokkum, H.M., J. Chem. Theory Comput., 2012, 49, 1-22.

88 S. Stoll and A. Schweiger, J. Magn. Reson., 2006, 178, 42-55.

89 M. J. T. Frisch, G.W. Schlegel, H.B. Scuseria, G.E. Robb, M.A. Cheeseman, J.R. Scalmani, G. Barone, V. Mennucci, B. Petersson, G.A.; et al., Gaussian 09, Revision D.01, 2009.

90 M. J. Frisch, G. W. Trucks, H. B. Schlegel, G. E. Scuseria, M. A. Robb, J. R. Cheeseman, G. Scalmani, V. Barone, G. A. Petersson, H. Nakatsuji, X. Li, M. Caricato, A. V. Marenich, J. Bloino, B. G. Janesko; et al., Gaussian 16, Wallingford, CT, 2016.

91 V. TURBOMOLE V7.1, 2016; Available from http://www.turbomole.com.

92 F. Neese, Wiley Interdiscip. Rev.: Comput. Mol. Sci. 2012, 2, 73-78.

93 F. Neese, ORCA-An Ab initio, Density Functional and Semiempirical Program Package.

94 B. de Souza, G. Farias, F. Neese and R. Izsák, J. Chem. Theory Comput., 2019, 15, 1896-1904.

95 F. Weigend and R. Ahlrichs, Phys. Chem. Chem. Phys., 2005, 7, 3297-3305.

96 F. Neese, J. Chem. Phys., 2005, 122, 034107. 